Pacific Journal of Mathematic 


\title{
FIXED POINT SETS OF 1-DIMENSIONAL PEANO CONTINUA
}

\author{
John R. Martin and E. D. Tymchatyn
}

\begin{abstract}
It is shown that every nonempty closed subset of a 1dimensional Peano continuum $X$ is the fixed point set of some continuous self-mapping of $X$.
\end{abstract}

1. Introduction. A topological space $X$ is said to have the complete invariance property (CIP) if every nonempty closed subset of $X$ is the fixed point set of some continuous self-mapping of $X$. The term CIP was suggested by L. E. Ward, Jr. in [5, p. 553] where it was asked if every Peano continuum had CIP. Examples have been given in [3], $[4,3.1]$ which show that $n$-dimensional Peano continua need not have CIP if $n>1$. In [4, 3.4] it is asked if every 1-dimensional Peano continuum has CIP. The purpose of this note is to answer that question in the affirmative by showing that every 1-dimensional Peano continuum has CIP.

2. Preliminaries, Let $M$ be a metric space. A sequence of subsets of $M$ is called a null sequence provided that for any $\varepsilon>0$ at most a finite number of its elements has diameter greater than $\varepsilon$. The space $M$ is said to have property $S$ provided that for each $\varepsilon>0$, $M$ is the union of a finite number of connected sets each of diameter less than $\varepsilon$. A partitioning of $M$ is a finite collection $\mathscr{Z}$ of pairwise disjoint connected open subsets of $M$ whose union is dense in $M$. If the mesh of $\mathscr{C}$ is less than $\varepsilon$ (each element of $\mathscr{C}$ is of diameter less than $\varepsilon$ ), $\mathscr{C}$ is called on $\varepsilon$-partitioning. A sequence $\mathscr{Q}_{1}$, $\mathscr{U}_{2}, \cdots$ of partitionings is called a decreasing sequence if, for each positive integer $i, \mathscr{U}_{i+1}$ is a refinement of $\mathscr{C}_{i}$ and the mesh of $\mathscr{U}_{i}$ approaches 0 as $i$ increases without limit. It is well-known [1, p. 545] that every Peano continuum has a decreasing sequence of partitionings.

A dendron is a connected, simply connected, finite graph. The closure of a subset $A$ of a topological space shall be denoted by $C 1(A)$.

\section{The result.}

Theorem Every 1-dimensional Peano continuum has the complete invariance property.

Proof. Let $X$ be a 1-dimensional Peano continuum and let $A$ be 
a closed subset of $X$. Let $\mathscr{C}_{1}, \mathscr{C}_{2}, \cdots$ be a decreasing sequence of $(1 / i)$-partitionings of $X$. Then each $\mathscr{C}_{i}$ is a finite collection of open connected pairwise disjoint sets of diameter less than $1 / i$ such that for each $i \bigcup\left\{U \mid U \in \mathscr{H}_{i}\right\}$ is dense in $X$ and $\mathscr{U}_{i+1}$ refines $\mathscr{H}_{i}$. We suppose

$$
\begin{aligned}
& \mathscr{C}_{1}=\left\{U_{1,1}, \cdots, U_{1, m_{0,1}}\right\} \\
& \mathscr{C}_{2}=\left\{U_{2, i, j} \mid U_{2, i, j} \subset U_{1, i} \in \mathscr{U}_{1} \text { and } j \in\left\{1, \cdots, m_{1, i}\right\}\right\}
\end{aligned}
$$

and for $i>1$

$$
\begin{aligned}
& \mathscr{U}_{i}=\left\{U_{i, j_{1}, \cdots, j_{i}} \mid U_{i, j_{1}, \cdots, j_{i}} \subset U_{i-1, j_{1}, \cdots, j_{i-1}} \in \mathscr{U}_{i-1}\right. \\
& \text { and } \left.j_{i} \in\left\{1, \cdots, m_{i-1, j_{1}}, \cdots, j_{i-1}\right\}\right\} \text {. }
\end{aligned}
$$

For each $i=1,2, \cdots$ let

$$
\mathscr{C}_{i}^{\prime}=\left\{U \in \mathscr{C}_{i} \mid C 1(U) \cap A \neq \varnothing\right\} .
$$

Without loss of generality,

$$
\begin{aligned}
\mathscr{U}_{1}^{\prime}=\left\{U_{1,1}, \ldots, U_{1, n_{0,1}}\right\} \quad \text { and for } i>1 \\
\mathscr{U}_{i}^{\prime}=\left\{U_{i, j_{1}, \cdots, j_{i}} \mid U_{i, j_{1}, \cdots, j_{i}} \subset U_{i-1, j_{1}}, \cdots, j_{i-1} \in \mathscr{U}_{i-1}^{\prime}\right. \\
\text { and } \left.j_{i} \in\left\{1, \cdots, n_{i-1, j_{1}, \cdots, j_{i-1}}\right\}\right\} .
\end{aligned}
$$

Notice that $A \subset C 1\left(\bigcup \mathscr{U}_{i}^{\prime}\right)$ for each $i$.

Let $A_{1,1}$ be an arc in $X$ which meets $U_{1,1}$ and $U_{1,2}$. If $A_{1,1} \cap U_{1,3} \neq \varnothing$ let $A_{1,2}=\varnothing$. If $A_{1,1} \cap U_{1,3}=\varnothing$ let $A_{1,2}$ be an arc such that $A_{1,2}$ meets $U_{1,3}$ and $A_{1,1} \cap A_{1,2}$ is an endpoint of $A_{1,2}$. Suppose $A_{1,1} \cup \cdots \cup A_{1, i}$ is a finite dendron such that $A_{1,1} \cup \cdots \cup A_{1, i}$ meets $U_{1, j}$ for each $j \in$ $\{1, \cdots, i+1\}$. If $i+2 \leqq n_{0,1}$ let $A_{1, i+1}=\varnothing$ if $\left(A_{1,1} \cup \cdots \cup A_{1, i}\right) \cap$ $U_{1, i+2} \neq \varnothing$, otherwise, let $A_{1, i+1}$ be an arc which meets $U_{1, i+2}$ and such that $\left(A_{1,1} \cup \cdots \cup A_{1, i}\right) \cap A_{i, i+1}$ is an endpoint of $A_{1, i+1}$. By induction $A_{1, i}$ is defined for each $i \in\left\{1, \cdots, n_{0,1}-1\right\}$. Let

$$
B_{1}=A_{1,1} \cup \cdots \cup A_{1, n_{0,1}-1} \text {. }
$$

Suppose $B_{1}, \cdots, B_{k}$ are finite dendrons such that $B_{1} \subset B_{2} \subset \cdots \subset B_{k}$, $B_{k}$ meets $U$ for each $U \in \mathscr{U}_{k}^{\prime}$ and

$$
B_{k}-B_{k-1} \subset \bigcup\left\{U \mid U \in \mathscr{Q}_{k}^{\prime}\right\} \text {. }
$$

For each $U_{k, j_{1}, \ldots, j_{k}} \in \mathscr{U}_{k}{ }^{\prime}$ let $A_{k+1, j_{1}, \cdots, j_{k}, 1}=\varnothing$ if $B_{k}$ meets $U_{k+1, j_{1}, \cdots, j_{k}, 1}$, otherwise, let $A_{k+1, j_{1}, \ldots, j, 1}$ be an arc in $U_{k, j_{1}, \ldots, j_{k}}$ which meets $U_{k+1, j_{1}, \cdots, j_{k},}$ and such that $B_{k} \cap A_{k+1, j_{1}, \cdots, j_{k}, 1}$ is an endpoint of $A_{k+1, j_{1}, \cdots, j_{k}, 1}$. Let $U_{k, j_{1}, \cdots, j_{k}} \in \mathscr{U}_{k}^{\prime}$ and suppose $A_{k+1, j_{1}, \cdots, j_{k}, i}$ is defined for $i \in\{1, \cdots, m\}$ where $m<n_{k, j_{1}, \cdots, j_{k}}$. If $B_{k} \cup \bigcup_{i=1}^{m} A_{k+1} j_{1}, \cdots, j_{k}, i$ meets $U_{k+1, j_{1}, \cdots, j_{k}, m+1}$ let $A_{k+1, j_{1}, \cdots, j_{k}, m+1}=\varnothing$, otherwise, let $A_{k+1, j_{1}, \ldots, j_{k}, m+1}$ be an arc in $U_{k, j_{1}, \cdots, j_{k}}$

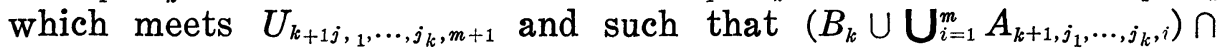
$A_{k+1, j_{1}, \ldots, j_{k}, m+1}$ is an endpoint of $A_{k+1, j_{1}, \ldots, j_{k}, m+1}$. Let 


$$
B_{k+1}=B_{k} \cup \bigcup\left\{A_{k+1} j_{1}, \ldots, j_{k}, j_{k+1} \mid U_{k+1} j_{1}, \ldots, j_{k}, j_{k+1} \in \mathscr{C}_{k+1}^{\prime}\right\} .
$$

By induction $B_{k}$ is defined for each $k=1,2, \cdots$.

Let $B=A \cup B_{1} \cup B_{2} \cup \cdots$. Then $B$ is connected since $B_{1} \subset B_{2} \subset \cdots$, each $B_{i}$ is connected and $\bigcup B_{i}$ is dense in $B$. The set $B$ is compact since $B-U$ is contained in a finite dendron for each open neighborhood $U$ of $A$ and $A$ is compact. It is easy to show that $B$ has property $S$. To see this, let $\varepsilon>0$ and let $n$ be a positive integer such that $3 / n<\varepsilon$. Since $B_{n}$ has property $S$, there is a positive integer $m$ and continua $K_{1}, \cdots, K_{m}$ such that $B_{n}=K_{1} \cup \cdots \cup K_{m}$ and each $K_{i}$ has diameter $\left\langle 1 / n\right.$. Let $U \in \mathscr{C}_{n}{ }_{n}$. Let $K_{i_{1}}, \cdots, K_{i_{r}}$ be the members of $\left\{K_{1}, \cdots, K_{m}\right\}$ which meet $U$. Then $\left(K_{i_{1}} \cup \cdots \cup K_{i_{r}} \cup U\right) \cap B$ has at most $i_{r}$ components, and each of these has diameter $<3 / n<\varepsilon$. It follows that $B$ has property $S$ and hence is locally connected (see [6, p. 20]). By [2, p. 174] $B$ is a retract of $X$.

It suffices to prove that there is a continuous mapping $f: B \rightarrow B$ such that $f(x)=x$ if and only if $x \in A$. Since $B$ is locally connected, each component of $B-A$ is open in $B$. Hence, $B-A$ has at most countably many components $C_{1}, C_{2}, \cdots$. Notice that every component of $B-A$ is a simply connected local graph. It follows from the last sentence and from the construction of the sets $B_{k}$ that every sequence of pairwise disjoint $\operatorname{arcs}$ in $B-A$ is a null sequence. Hence, the sequence $C_{1}, C_{2}, \cdots$ is null. It suffices to prove, therefore, that for each $i \geqq 1$ there exists a continuous mapping $g_{i}: C 1\left(C_{i}\right) \rightarrow C 1\left(C_{i}\right)$ such that $g_{i}(x)=x$ if and only if $x \in C 1\left(C_{i}\right)-C_{i}$. The existence of $g_{i}$ follows easily from the fact that $C_{i}$ is a simply connected local graph in which every sequence of pairwise disjoint ares is null.

\section{REFERENCES}

1. R. H. Bing, Partitioning continuous curves, Bull. Amer. Math. Soc., 58 (1952), 536-556.

2. S. T. Hu, Theory of Retracts, Wayne State University Press, Detroit, 1965.

3. J. R. Martin, Fixed point sets of Peano continua, Pacific J. Math., 74 (1978), 163-166.

4. J. R. Martin and S. B. Nadler, Jr., Examples and questions in the theory of fixed point sets, Canad. J. Math., 31 (1979), 1017-1032.

5. L. E. Ward, Jr., Fixed point sets, Pacific J. Math., 47 (1973), 553-565.

6. G. T. Whyburn, Analytic Topology, Amer. Math. Soc., Colloquium Publications, Vol. 28, Providence, 1942.

Received February 23, 1979 and in revised form July 5, 1979. The first author's research was supported in the part by the National Research Council of Canada (Grant A8205), and the second author's research was supported in part by the National Research Council of Canada (Grant A5616).

University of Saskatchewan

Saskatoon, Saskatchewan

CANADA S7N 0W0 



\section{PACIFIC JOURNAL OF MATHEMATICS}

\section{EDITORS}

DONALD BABBITT (Managing Editor)

University of Galifornia

Los Angeles, California 90024

Hugo RossI

University of Utah

Salt Lake City, UT 84112

C. C. MoOre AND ANDrew OGG

University of California

Berkeley, CA 94720

\section{J. DugundjI}

Department of Mathematics University of Southern California

Los Angeles, California 90007

R. Finn and J. Milgram

Stanford University

Stanford, California 94305

ASSOCIATE EDITORS

E. F. Beckenbachi

B. H. NeumanN

F. WOLF

K. YOSHIDA

\section{SUPPORTING INSTITUTIONS}

UNIVERSITY OF BRITISH COLUMBIA

CALIFORNIA INSTITUTE OF TECHNOLOGY

UNIVERSITY OF CALIFORNIA

MONTANA STATE UNIVERSITY

UNIVERSITY OF NEVADA, RENO

NEW MEXICO STATE UNIVERSITY

OREGON STATE UNIVERSITY

UNIVERSITY OF OREGON
UNIVERSITY OF SOUTHERN CALIFONIA

STANFORD UNIVERSITY

UNIVERSITY OF HAWAII

UNIVERSITY OF TOKYO

UNIVERSITY OF UTAH

WASHINGTON STATE UNIVERSITY

UNIVERSITY OF WASHINGTON

Printed in Japan by International Academic Printing Co., Ltd., Tokyo, Japan 


\section{Pacific Journal of Mathematics \\ Vol. 89 , No. 1 \\ May, 1980}

David Bressoud, A note on gap-frequency partitions ................ 1

John David Brillhart, A double inversion formula ................. 7

Frank Richard Deutsch, Günther Nürnberger and Ivan Singer, Weak

Chebyshev subspaces and alternation .......................

Edward Richard Fadell, The relationship between Ljusternik-Schnirelman

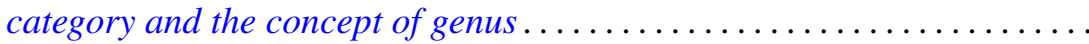

Harriet Jane Fell, On the zeros of convex combinations of polynomials.......

John Albert Fridy, An addendum to: "Tauberian theorems via block

dominated matrices" ..................................

Andrzej Granas, Ronald Bernard Guenther and John Walter Lee, Applications of topological transversality to differential equations. I. Some nonlinear

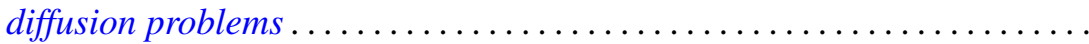

David E. Handelman and G. Renault, Actions of finite groups on self-injective

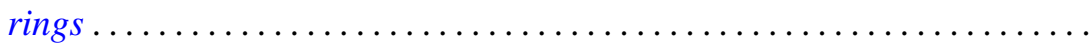

Michael Frank Hutchinson, Local $\Lambda$ sets for profinite groups .............

Arnold Samuel Kas, On the handlebody decomposition associated to a

Lefschetz fibration...

Hans Keller, On the lattice of all closed subspaces of a Hermitian space.....

P. S. Kenderov, Dense strong continuity of pointwise continuous

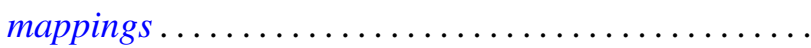

Robert Edward Kennedy, Krull rings.................

Jean Ann Larson, Richard Joseph Laver and George Frank McNulty,

Square-free and cube-free colorings of the ordinals ...

Viktor Losert and Harald Rindler, Cyclic vectors for $L^{p}(G)$

John Rowlay Martin and Edward D. Tymchatyn, Fixed point sets of

1-dimensional Peano continua...

Augusto Nobile, On equisingular families of isolated singularities ...

Kenneth Joseph Prevot, Imbedding smooth involutions in trivial bundles ...

Thomas Munro Price, Spanning surfaces for projective planes in four space.

Dave Riffelmacher, Sweedler's two-cocycles and Hochschild cohomology....

Niels Schwartz, Archimedean lattice-ordered fields that are algebraic over

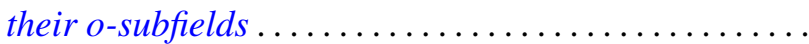

Chao-Liang Shen, A note on the automorphism groups of simple dimension groups.

Kenneth Barry Stolarsky, Mapping properties, growth, and uniqueness of

Vieta (infinite cosine) products ...

Warren James Wong, Maps on simple algebras preserving zero products. I.

The associative case ............................. 